American Journal of Infectious Diseases 7 (3): 80-82, 2011

ISSN 1553-6203

(C) 2011 Science Publications

\title{
Stimulatory Effects of Dihydroartemisinin on the Leucocyte Population of Wistar Albino Rats
}

\author{
${ }^{1}$ Utoh-Nedosa, U.A., ${ }^{2}$ P.A. Akah, ${ }^{2}$ C.O. Okoli, ${ }^{3}$ T. Ojemudia, ${ }^{4}$ I. Nedosa, \\ ${ }^{3}$ O.N. Adeyanju, ${ }^{5}$ K.S. Nedosa and ${ }^{6}$ I.K. Onyedibe \\ ${ }^{1}$ Department of Pharmacology and Toxicology, Faculty of Pharmaceutical Sciences, \\ Nnamdi Azikiwe University, P. M. B. 5025, \\ Awka, Anambra State, Nigeria \\ ${ }^{2}$ Department of Pharmacology and Toxicology, \\ Faculty of Pharmaceutical Sciences \\ University of Nigeria, Nsukka, Enugu State, Nigeria \\ ${ }^{3}$ Department of Parasitology, \\ Federal College of Veterinary and Medical Laboratory Technology, \\ Vom, Plateau State, Nigeria \\ ${ }^{4}$ Department of Industrial Microbiology, \\ Federal University of Technology, Owerri, Imo State, Nigeria \\ ${ }^{5}$ Evangelical Churches of West Africa Hospital (ECWA), \\ Egbe, P. M. B., Kogi State, Nigeria \\ ${ }^{6}$ Department of Medical Microbiology, \\ University of Jos Teaching Hospital, P.M. B. 2076, Jos, Plateau State, Nigeria
}

\begin{abstract}
Problem statement: Five dosages of Dihydroartemisinin (DHA) which included $1 \mathrm{mg} \mathrm{kg}^{-1}$; a repeated dose of $1 ; 2 ; 60$ and $80 \mathrm{mg} \mathrm{kg}^{-1}$ were administered orally to albino rats for 5 days. The results of the study showed that dihydroartemisinin treatment significantly elevated the total white cell count $(p<0.01)$; the percentage neutrophil count $(p<0.01)$ and the percentage lymphocyte count $(\mathrm{p}<0.05)$. It also increased the percentage monocyte count though its increase was not statistically significant. Approach: By increasing the population of neutrophils, lymphocytes and monocytes (which engage in chemo tactic response; microbial killing; microbial ingestion and antibody production) in the blood, dihydroartemisinin demonstrated that it stimulates increase in their population and uses them as part of its own arsenals of warfare against endoparasites (like malaria parasites) and pathogens. Results: The results of this study show that dihydroartemisinin stimulated a lot of new white blood cell production by haemopoetic sites of the body The increases in the total white blood cell count; the percentage neutrophils count and the percentage lymphocyte counts were significant at $p<0.01$ level of significance. Conclusion: The results of the study suggest that the phagocytic and immunological activities of the body's white blood cell population are important components of the efficacious Plasmodium Schizonticidal actions of Dihydroartemisinin in malaria treatment.
\end{abstract}

Key words: Dihydroartemisinin (DHA), cell count, dihydroartemisinin demonstrated, malaria treatment, microbial ingestion, antibody production, haemopoesis stimulation, laboratory procedures, dosage regimen, rapid therapeutic effect

\section{INTRODUCTION}

The artemisinin antimalarials have been found to be very efficacious and effective in the management of uncomplicated and complicated malaria (Asawamahaskda et al., 1994) The Artemisinin Combination Therapy
(ACT) drugs were seen by World Health Organization to be capable of offering new hope for the control of malaria because they have a rapid therapeutic effect in reducing the malaria parasite and diminishing symptoms.

Parasiticidal action of artemisinin has been suggested to occur through their engagement of the

Corresponding Author: Utoh-Nedosa, U.A., Department of Pharmacology and Toxicology, Faculty of Pharmaceutical Sciences, Nnamdi Azikiwe University, P. M. B. 5025, Awka, Anambra State, Nigeria 
parasite as an endooperoxide. In the process of this engagement the drug accumulates many times more in the infected cell than elsewhere just like quinacrine which was found to accumulate 500 times more in infected areas. Malaria parasite is suggested to be killed by alkylation of the parasite proteins by the drug.

This study investigated the effects of Dihydroartemisinin (DHA) on the blood cell population of albino rats.

\section{MATERIALS AND METHODS}

Dihydroartemisinin (DHA) was administered by oral intubation to 5 Adult test Wistar albino rats which weighed 106-140 g in 4 different treatments. Four adult Wistar rats of 106-140 gm weight served as controls to these test rats DHA was given daily at doses of $1 \mathrm{mg} \mathrm{kg}^{-1}$ (with a $2 \mathrm{mg} \mathrm{kg}^{-1}$ starting dose), 2, 60 and $80 \mathrm{mg} \mathrm{kg}^{-1}$ Dihydroartemisinin (DHA), to 4 sets of test rats for 5 days.

Four young adult wistar albino rats weighing 75-90 $\mathrm{g}$ were given the $11 \mathrm{mg} \mathrm{kg}^{-1}$ DHA (with a $2 \mathrm{mg} \mathrm{kg}^{-1}$ starting dose) also by intubation for 5 days and the same dosage regimen was repeated to them after a one week interval (repeat treatment). Four rats which weighed 75-90 $\mathrm{g}$ and were of the same age as the tst rats served as controls to this group.

The control rats in each of the 5 experiments were given distilled water equivalents of the dosage of DHA taken by their test group in place of dihydroartemisinin, also by oral intubation.

The test and control rats were weighed $10 \mathrm{~m} 1$ nutes before the administration of the first dose of a tested dosage regimen and 10 m1nutes after the administration of the last dose of that dosage regimen.

The test and control rats were sacrificed $24 \mathrm{~h}$ after the administration of the last dose of each tested dosage regimen of dihydroartemisinin.

The blood of the sacrificed test and control rats was collected through the subclavian artery into EDTA anticoagulanted blood collection bottles before each animal was sacrificed. The total and deferential white blood cell counts of the blood of the test and control rats were done using standard laboratory procedures.

\section{RESULTS}

The results of our study showed that the total white blood cell count, the percentage neutrophil count and the percentage lymphocyte counts were elevated in the DHA treated rats. DHA treatment significantly $(p<0.01)$ elevated the total blood cell count. The results of the differential leucocyte counts showed that DHA significantly elevated the percentage neutrophil count $(\mathrm{p}<0.01)$ and the percentage lumphocyte count $(\mathrm{p}<0.01, \mathrm{p}<0.05)$. DHA treatment also increased the percentage monocyte count; did not have much effect on the percentage eosinophil count and had no effect at all on the basophil count.

These effects of DHA on the total white blood cells (on the total and differential WBC counts) were DHA concentration and DHA dose repetition and time dependent.

\section{DISCUSSION}

The results of this study show that dihydroartemisinin stimulated a lot of new white blood cell production by haemopoetic sites of the body (Fig. 1-3).

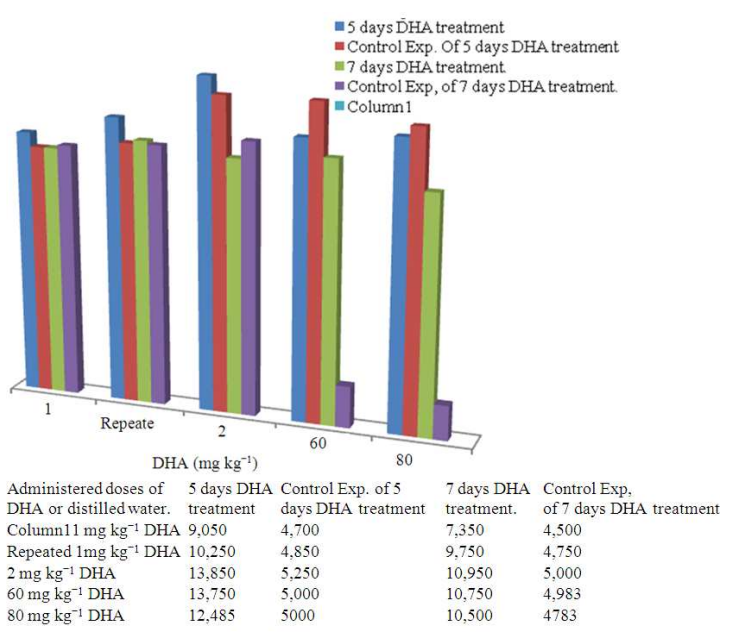

Fig. 1: The Effect of Dihydroartemisinin treatment on the total white blood cell count in $\mathrm{mm}^{3}$

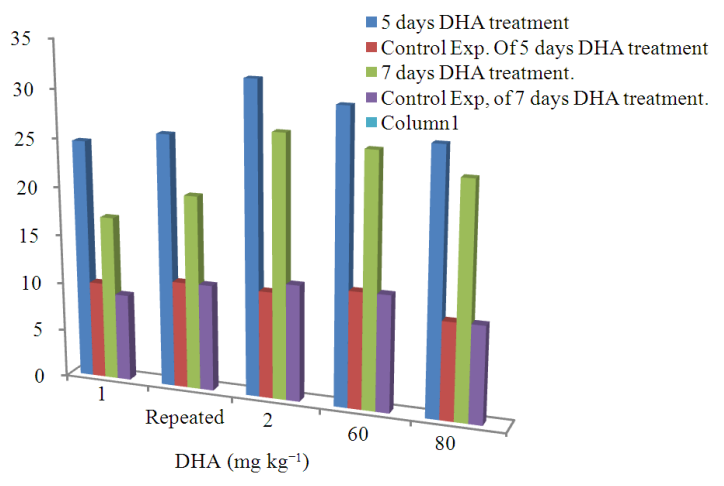

Fig. 2: The Effect of Dihydroartemisinin treatment on the percentage neutrophils count [of the WBC] of Dihydroartemisinin-treated and Control albino rats 


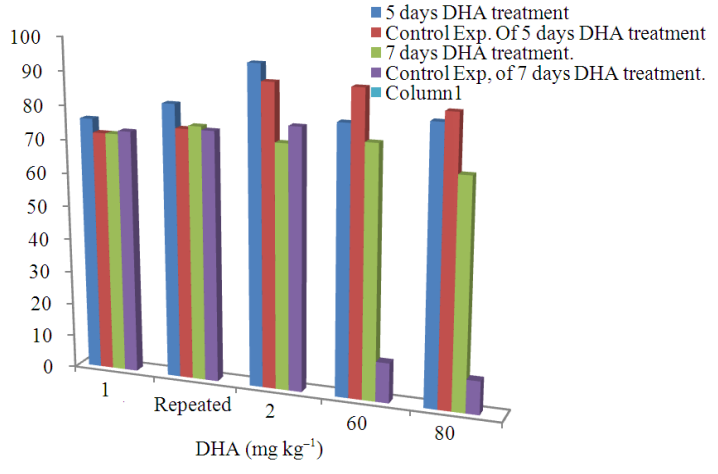

Fig. 3: The Effect of Dihydroartemisinin treatment on percentage lymphocyte count [of the WBC] of Dihydroartemisinin treated and Control albino rats

The increases in the total white blood cell count; the percentage neutrophils count and the percentage lymphocyte counts were significant at $p<0.01$ level of significance which shows that the haemopoesis stimulation effect of DHA was very potent. These effects of dihydroartemisinin on haemopoesis confirm the observation in a previous that the action of artemisinins as antimalarials is linked io their action on intrcellular hemin (Meshnick et al., 1994; Ponmee et al., 2007).

Since the white blood cells constitute the disease fighting army of the body, this potent white blood cell population explosion effect of dihydroartemisinin on the blood is a very important aspect of its parasite fighting and elimination strategies as dihydroartemisinin is $90 \%$ bound to plasma proteins. The events that take place in the blood during clinical malaria are the hallmark of malaria (Blacklock et al., 1973). The role of dihydroartemisinin in interacting with blood producing cells is significant in malaria as dihydroartemisinin has been found to interact with proteins (Asawamahaskda et al., 1994).

\section{CBONCLUSION}

The findings of this study suggest that the phagocytic and immunological activities of the white blood cells (especially those of neutrophils and lymphocytes) constitute a component of the plasmodium killing actions of dihydroartemisinin in malaria treatment. This finding is very important as the mechanisim of antimalarial action of artemisinin is unsettled (Robert and Meunier, 1998).

\section{REFERENCES}

Asawamahaskda, W., I. Ittratt, Y.M. Pu, H. Ziffer and S.R. Meshnick, 1994. Reaction of antimalarial endoperoxides with specific parasite proteins. Antimicrob. Agents Chemother., 38: 1854-1858. PMCID: PMC284649

Blacklock, D.B., T. Southwell, T.H. Davey and W. Crewe, 1973. A Guide to Human Parasitology for Medical Practitioners. 9th Edn., H.E. Lewis Co Ltd., London, pp: 218.

Meshnick, S.R., T.E. Taylor and P. Kamchonwongpaisan 1996. Artemisinin and the antimalarial endoperoxides: From herbal remedy to targeted chemotherapy. Microbiol. Rev., 60: 301315. PMID: 8801435

Ponmee, N., T. Chuchue, P. Wilairat, Y. Yuthavong and S. Kamchonwonpaisan, 2007. Artemisinin effectiveness in erythrocytes is reduced by heme and heme-containing proteins. Biochem. Pharmacol., 74: 153-160. PMID: 17498668

Robert, A. and B. Meunier, 1998. Is alkylation the main mechanism of action of the antimalarial drug artemisinin? Chem. Soc. Rev., 27: 273-280. 